Revue musicale OICRM

\title{
Stratégies pédagogiques visant le développement des habiletés métacognitives du musicien en formation afin d'optimiser l'efficacité de ses pratiques instrumentales
}

\section{Malinalli Peral Garcia et Francis Dubé}

Volume 1, numéro 1, 2012

Profession musicale au Québec

URI : https://id.erudit.org/iderudit/1055859ar

DOI : https://doi.org/10.7202/1055859ar

Aller au sommaire du numéro

Éditeur(s)

OICRM

ISSN

2368-7061 (numérique)

Découvrir la revue

Citer cet article

Peral Garcia, M. \& Dubé, F. (2012). Stratégies pédagogiques visant le développement des habiletés métacognitives du musicien en formation afin d'optimiser l'efficacité de ses pratiques instrumentales. Revue musicale OICRM, 1(1), 74-89. https://doi.org/10.7202/1055859ar
Résumé de l'article

Cet article porte sur les connaissances et les stratégies pédagogiques à mettre en action afin de développer les habiletés métacognitives du musicien en formation. Nous expliquons d'abord les diverses composantes de la métacognition que le professeur d'instrument doit intégrer à son acte pédagogique pour guider l'élève sur le plan métacognitif. Nous exposons ensuite les modes d'intervention pédagogique à privilégier pour engager l'élève musicien dans une démarche métacognitive. Puis, nous proposons deux stratégies pédagogiques issues des sciences éducatives élaborées pour développer les habiletés métacognitives de l'élève, stratégies que nous avons adaptées aux besoins spécifiques de la pratique instrumentale. Ces outils offrent au musicien pédagogue des référents pour guider le développement des habiletés métacognitives de ses élèves. 


\title{
Stratégies pédagogiques visant le développement des habiletés métacognitives du musicien en formation afin d'optimiser l'efficacité de ses pratiques instrumentales
}

\author{
Malinalli Peral Garcia \\ et Francis Dubé
}

\begin{abstract}
Résumé
Cet article porte sur les connaissances et les stratégies pédagogiques à mettre en action afin de développer les habiletés métacognitives du musicien en formation. Nous expliquons d'abord les diverses composantes de la métacognition que le professeur d'instrument doit intégrer à son acte pédagogique pour guider l'élève sur le plan métacognitif. Nous exposons ensuiteles modes d'intervention pédagogique à privilégier pour engager l'élève musicien dans une démarche métacognitive. Puis, nous proposons deux stratégies pédagogiques issues des sciences éducatives élaborées pour développer les habiletés métacognitives de l'élève, stratégies que nous avons adaptées aux besoins spécifiques de la pratique instrumentale. Ces outils offrent au musicien pédagogue des référents pour guider le développement des habiletés métacognitives de ses élèves.

Mots clés : enseignement instrumental ; métacognition ; pratique instrumentale ; répétition instrumentale; stratégies pédagogiques.
\end{abstract}

\footnotetext{
Abstract

This article is about educational knowledge and strategies that are to be used in developing the metacognitive skills of the musician in training. We first explain the metacognition's various components that the teacher must include in his/ her teaching to guide the student on the metacognitive level. We then describe the methods of educational intervention that should be favored in order to engage the student musician in a metacognitive approach. Afterwards, we propose two educational strategies derived from educational sciences and created to develop the metacognitive skills of the student. These strategies have been adapted to the specific needs of instrumental practice. These tools offer to the musician-teacher some referents to guide the development of the metacognitive skills of his/her students.

Keywords: educational strategies; instrumental practice; instrumental repetition; instrumental teaching; metacognition.
} 
Selon Flavell (1976, p. 232), la métacognition concerne les connaissances qu'une personne possède sur ses processus et produits cognitifs. Celles considérées expertes dans leur domaine, que ce soit en musique ou dans d'autres secteurs professionnels, démontrent davantage d'habiletés métacognitives que les novices (Chase et Simon 1973; Ericsson, Krampe et Tesch-Romer 1993 ; Hallam 2001). En musique, ce type d'habiletés joue un rôle fondamental dans l'acquisition et le développement d'habiletés musicales, car elles permettent notamment à l'instrumentiste de répéter plus efficacement durant ses séances de travail (Hallam 2001 ; Barry et Hallam 2002, p. 154-155).

En effet, les musiciens professionnels organiseraient leurs séances de répétition en fonction des erreurs détectées dans le but de mieux les corriger (Hallam 1997, cité par Woody 2001, p. 11), tandis que les musiciens en formation, particulièrement les débutants, passeraient jusqu'à 90\% de leur travail instrumental à jouer leur pièce du début jusqu'à la fin, sans utiliser de stratégies spécifiques visant à améliorer leur performance (McPherson et Renwick 2001, p. 174). Or, c'est précisément l'acquisition d'habiletés métacognitives qui amène l'instrumentiste à surveiller et à corriger plus efficacement ses propres erreurs (Kenny 1992, cité par Woody 2001, p. 11). Vu leur importance dans le cadre du travail instrumental, il serait primordial de développer les habiletés métacognitives de l'élève musicien afin d'accroître la qualité de ses répétitions.

Sur le plan pédagogique, le professeur d'instrument peut jouer un rôle central dans le développement des habiletés métacognitives de l'élève. Pour remplir ce rôle, il doit amener l'élève à réfléchir sur la façon de s'exercer, d'apprendre et de mémoriser son répertoire, en insistant tout particulièrement sur la manière dont l'apprenant peut intégrer cette approche réflexive à ses connaissances musicales (Aiello et Williamon 2002, p. 176). En revanche, pour travailler dans cette direction, l'enseignant doit posséder certaines qualités et stratégies pédagogiques liées spécifiquement au développement des habiletés métacognitives de l'élève (Lafortune, Jacob et Hébert 2000, p. 39-54 ; Doly 1997, p. 45-67), et c'est essentiellement le sujet dont nous traiterons dans le cadre de cet article.

Pour être plus précis, nous définirons d'abord ce qu'est la métacognition et nous expliquerons ses différentes composantes. Ensuite, nous exposerons les habiletés métacognitives que l'on observe le plus souvent chez le musicien expert, dans le but de les mettre en relation avec celles devant être développées chez le musicien en formation. Enfin, nous proposerons des modes d'intervention et des stratégies pédagogiques pouvant contribuer au développement des habiletés métacognitives de l'élève musicien.

\section{DÉFINITION ET COMPOSANTES DE LA MÉTACOGNITION}

Depuis le début du $\mathrm{xx}^{\mathrm{e}}$ siècle, plusieurs auteurs ont réalisé des recherches scientifiques ainsi que des écrits théoriques sur les processus liés à la métacognition ${ }^{1}$.

1 Pour plus d'informations, consulter Brown (1987). Cette auteure présente un bref historique entourant le concept de métacognition. 
Toutefois, Flavell est souvent considéré comme le pionnier des recherches sur la métacognition (Saint-Pierre 1994, p. 531), car ses études sur la méta-mémoire ${ }^{2}$ 1'ont $^{\prime}$ amené à proposer la première définition de ce concept. Selon lui, la métacognition

fait référence à la connaissance qu'une personne a de ses propres processus et produits cognitifs ou de toute autre chose qui s'y rapporte... Par exemple, je fais de la métacognition [...] si je remarque que j'ai plus de difficulté à apprendre A que B ; si cela me frappe que je doive revérifier $C$ avant de l'accepter comme un fait [...] La métacognition fait référence, entre autres choses, à la surveillance active et à la régulation subséquente et à l'orchestration de ces processus... habituellement afin d'atteindre quelque but ou objectif concret (Flavell 1976, p. 232) ${ }^{3}$.

De plus, la métacognition reposerait sur deux grandes composantes principales, soit les connaissances métacognitives (Flavell 1979, p. 906 ; 1987, p. 21) ainsi que l'utilisation et l'adaptation de ces connaissances à la gestion des processus mentaux (Brown 1987, p. 67).

\section{Les connaissances métacognitives}

Les connaissances métacognitives sont regroupées en trois volets principaux, soit les connaissances sur les personnes, sur les tâches et sur les stratégies.

\section{Connaissances sur les personnes}

Les connaissances sur les personnes se divisent en trois sous-catégories : intraindividuelles, interindividuelles ou universelles (Flavell 1987, p. 22-23). Les intraindividuelles concernent les connaissances ou les croyances que l'individu possède sur son propre fonctionnement cognitif. Par exemple, savoir qu'il a de la difficulté à lire la musique contemporaine ou que son travail instrumental s'avère plus efficace lorsqu'il répète le matin plutôt que le soir, entre dans cette catégorie.

Les connaissances interindividuelles concernent celles où l'individu se compare à d'autres personnes. Par exemple, lorsqu'un musicien est conscient qu'il arrive à mieux jouer le répertoire virtuose que plusieurs musiciens de sa génération, il s'agit d'une connaissance interindividuelle.

Les connaissances universelles portent sur les représentations qu'a l'individu sur des aspects généraux de la cognition humaine. Il peut s'agir, entre autres, de connaissances ou d'intuitions liées au fonctionnement de la pensée, de la mémoire ou de l'in-

2 Flavell a été le premier à utiliser le terme de « méta-mémoire » en 1971. Voir Flavell 1971, p. 277.

3 " [...] refers to one's knowledge concerning one's own cognitive processes and products or anything related to them... For example, I am engaging in metacognition [...] if I notice that I am having more trouble learning $A$ than B; if it strikes me that I should double check C before accepting it as a fact [...] Metacognition refers, among other things, to the active monitoring and consequent regulation and orchestration of these processes [...] usually in the service of some concrete goal or objective » (Flavell 1976, p. 232). 
telligence. À titre d'exemple, savoir que la mémoire à court terme peut traiter environ sept éléments à la fois (+ ou - 2) à partir de l'âge de 14 ans.

2. Connaissances sur les tâches

Les connaissances sur les tâches portent sur la nature et les exigences de celles-ci quant à leur réalisation. Il s'agit de connaissances acquises au sujet de différentes informations liées aux expériences antérieures et sur le traitement que chacune d'entre elles nécessite pour être mise en œuvre (Flavell 1987, p. 22-23). Un exemple entrant dans cette catégorie de connaissances serait que le musicien doit être conscient qu'il existe quatre types de mémoire intervenant dans le processus de mémorisation d'une pièce - auditive, visuelle, kinesthésique, conceptuelle - mais qu'il est préférable d'axer l'apprentissage mnémonique sur l'utilisation de la mémoire conceptuelle, car ce type de mémoire aide l'instrumentiste à se sentir plus rassuré au moment de jouer sans la partition (Dubé 2006, p. 19).

\section{Connaissances sur les stratégies}

Les connaissances sur les stratégies concernent les différentes procédures à utiliser afin d'atteindre un objectif précis. Selon l'objectif visé, ces stratégies peuvent être classées en stratégies cognitives et en stratégies métacognitives. Celles dites cognitives permettent à l'individu d'atteindre un but cognitif, par exemple, lire une partition afin de la déchiffrer. Quant aux stratégies métacognitives, elles visent à vérifier si le résultat obtenu est satisfaisant ou non, par exemple, réviser la même partition en utilisant une stratégie permettant de vérifier qu'aucune fausse note n'est apprise.

Selon Flavell (1987, p. 23), les connaissances sur les personnes, les tâches et les stratégies sont toujours en interaction entre elles et les interactions liées à ces connaissances ou intuitions sont apprises de la même façon que les connaissances métacognitives. Par ailleurs, les connaissances métacognitives ne sont pas différentes des autres types de connaissances emmagasinées dans la mémoire à long terme, car elles peuvent être activées volontairement (Flavell 1979, 907-908). En revanche, elles peuvent aussi être activées automatiquement et involontairement lors de l'activité cognitive, et ce de manière plus ou moins consciente.

D'après Brown (1987, p. 67-68), les connaissances métacognitives sont relativement stables et verbalisables, c'est-à-dire que la personne peut exprimer à haute voix ce qu'elle croit à propos de son processus d'apprentissage, des stratégies s'avérant les plus efficaces, etc. Cependant, ces connaissances peuvent être vraies ou erronées. Par exemple, si une personne croit qu'elle doit relier les nouvelles connaissances en apprentissage à celles déjà emmagasinées dans la mémoire à long terme (connaissance métacognitive universelle sur les personnes) pour assimiler efficacement un concept, il s'agit d'une connaissance avérée. Toutefois, si elle croit que pour développer la vitesse d'une pièce, le musicien doit toujours s'exercer en augmentant graduellement la vitesse du geste (connaissance métacognitive sur les stratégies), il s'agit, dans ce cas, d'une connaissance erronée (Jørgensen 2004, p. 94). De plus, les connaissances métacognitives se développeraient avec l'âge. Ce type de connaissances requiert que les apprenants prennent du recul et considèrent leurs propres processus cognitifs comme des objets de pensée et de réflexion (Brown 1987, p. 68). 


\section{Les expériences métacognitives}

Flavell (1979, p. 908-909) affirme que les connaissances métacognitives peuvent engendrer des expériences cognitives ou affectives conscientes qu'il nomme expériences métacognitives. Ces expériences se distinguent des autres types d'expériences car elles ont un lien spécifique avec l'activité intellectuelle (Flavell 1987, p. 24). À titre d'exemple, quand une personne est consciente qu'elle ressent un sentiment d'anxiété lorsqu'elle n'arrive pas à comprendre quelque chose qu'elle désire intégrer à son savoir, il s'agit alors d'une expérience métacognitive. De plus, la personne apprend avec l'âge à interpréter ce type d'expériences et à y répondre adéquatement, contrairement aux jeunes enfants qui les vivent souvent de façon consciente, sans pour autant être en mesure de les interpréter correctement (ibid.).

En outre, Flavell (ibid., p. 28) explique que l'individu est probablement plus susceptible de vivre des expériences métacognitives lorsque :

- la situation le demande explicitement;

- la situation n'est ni complètement nouvelle, ni complètement familière pour lui, c'est-à-dire lorsque la personne connaît suffisamment la situation pour être perplexe et pour formuler des questions, mais insuffisamment pour que le processus soit entièrement automatique et sans effort ;

- la personne fait des inférences, porte des jugements ou prend des décisions correctes ;

- l'activité cognitive se retrouve au cœur d'une résolution de problème ;

- l'attention et les ressources mnémoniques de la personne ne sont pas amoindries par d'autres expériences subjectives plus urgentes, comme la peur, l'anxiété ou la dépression.

Les expériences métacognitives jouent également un rôle important dans le processus d'apprentissage car, toujours selon Flavell (1979, p. 908), elles peuvent avoir un effet décisif sur les tâches ou sur les objectifs cognitifs poursuivis, ainsi que sur les connaissances et sur les stratégies employées. De façon plus précise, lorsque l'individu vit une expérience métacognitive, il peut établir de nouveaux objectifs, de même que réviser ou abandonner ceux établis au départ. Ce type d'expériences peut même affecter sa base de connaissances métacognitives. Par exemple, le musicien qui expérimente de l'anxiété lorsqu'il réalise que sa façon de s'exercer ne l'amène pas aux résultats désirés (expérience métacognitive) risque de changer de stratégies de travail afin de mieux y parvenir. En fait, la mobilisation des connaissances métacognitives en interaction avec les expériences métacognitives donnerait lieu à la gestion des processus mentaux.

\section{La gestion des processus mentaux}

La deuxième principale composante de la métacognition concerne la gestion des processus mentaux. Elle correspond à ce que Lafortune, Jacob et Hébert (2000) appellent des « habiletés métacognitives ». Pour ces auteurs :

une habileté métacognitive est cette capacité à mobiliser ses connaissances et savoir-faire, mais dans l'intention explicite de planifier l'exécution d'une 
tâche afin de mieux la superviser, l'évaluer et porter un jugement critique sur l'efficacité de sa démarche au regard des stratégies mises en place et du but poursuivi. Ce jugement permet non seulement d'enrichir ses connaissances métacognitives, mais de développer un savoir conscient qui peut être déployé dans des situations de plus en plus complexes. Les principales manifestations d'une habileté métacognitive sont le contrôle et la régulation de la démarche d'apprentissage parce qu'ils sont le résultat d'une évaluation constante et consciente et en favorisent ainsi la réutilisation (Lafortune, Jacob et Hébert 2000, p. 12-13).

Ainsi, les habiletés métacognitives permettent à l'individu d'utiliser et d'adapter ses propres connaissances métacognitives afin de gérer son activité mentale. Elles comportent trois types d'activités en lien avec l'exécution d'une tâche, soit des activités de planification, de contrôle et de régulation.

\section{Les activités de planification}

Les activités de planification consistent à établir des objectifs et à choisir des stratégies en fonction des objectifs d'abord identifiés, ainsi qu'à déterminer les paramètres d'évaluation à utiliser pendant et à la fin de l'exécution de la tâche. À titre d'exemple, un musicien utilise des activités de planification lorsqu'il décide, au début d'une séance de travail instrumental, comment résoudre un passage technique à l'aide de stratégies spécifiques, détermine combien de temps il devra travailler ce passage pour y arriver, et lorsqu'il prévoit la manière dont il devra s'autoévaluer durant et à la fin de la répétition afin d'atteindre son objectif.

\section{Les activités de contrôle}

Les activités de contrôle visent à surveiller et à vérifier l'efficacité de l'activité cognitive en cours. Elles consistent principalement à évaluer les actions entreprises pendant l'apprentissage. Se demander si la stratégie choisie pour maîtriser une difficulté instrumentale donne les résultats escomptés est un exemple d'activité de contrôle.

\section{Les activités de régulation}

Les activités de régulation consistent à modifier la démarche utilisée pendant l'activité cognitive après avoir mis en œuvre les activités de contrôle. Il peut s'agir, par exemple, de conserver la stratégie choisie en début de répétition pour résoudre une difficulté technique, mais de diminuer la vitesse du geste initialement prévue afin de la régler plus efficacement.

Selon Brown (1987, p. 68), les activités de planification, de contrôle et de régulation sont relativement instables puisqu'elles varient en fonction de la tâche à effectuer et de la situation d'apprentissage dans laquelle elle est réalisée. De plus, elles ne sont pas toujours verbalisables car le fait de savoir comment faire quelque chose ne signifie pas nécessairement que la personne réalise l'activité consciemment, ni qu'elle puisse 
l'exprimer avec des mots. Ces activités sont aussi relativement indépendantes de l'âge puisque tout apprentissage actif demande une forme d'autorégulation. En effet, les jeunes enfants régulent et surveillent, tout comme les adultes, leurs propres activités d'apprentissage. La figure 1 offre, à titre de résumé, une vue synthétisée des notions métacognitives expliquées jusqu'à maintenant.

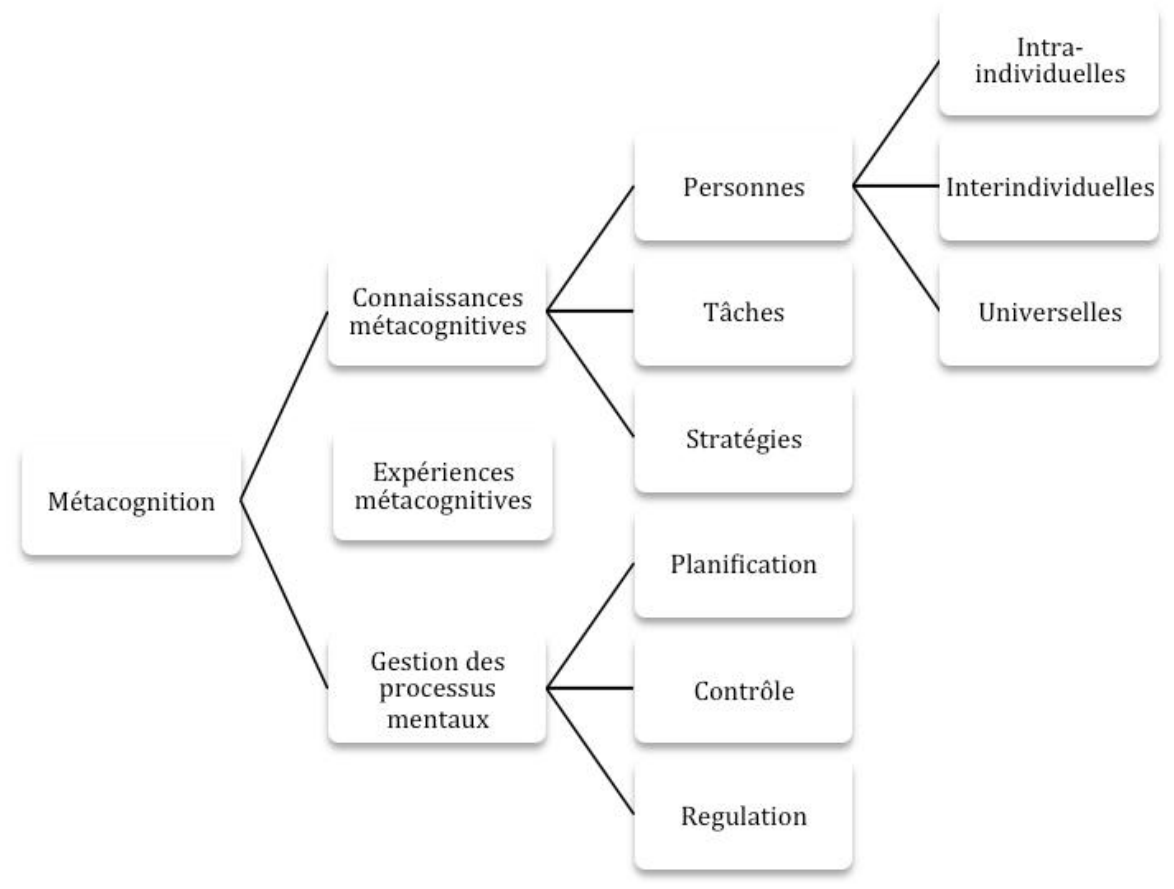

Figure 1 : Notions métacognitives.

\section{LE MUSICIEN EXPERT ET L’ENSEIGNANT GUIDE DE LA MÉTACOGNITION}

Habiletés métacognitives du musicien expert

Des recherches portant sur le développement de l'expertise suggèrent que les personnes possédant une expérience considérable dans un domaine spécifique possèdent beaucoup plus d'habiletés métacognitives que les novices (Chase et Simon 1973 Ericsson, Krampe et Tesch-Romer 1993 ; Hallam 2001). En effet, les experts démontrent une capacité d'analyse leur permettant d'identifier plus facilement et rapidement les problèmes à régler (Hallam 2001, p. 28). De plus, ils adaptent leurs façons de faire aux contraintes inhérentes à la tâche à effectuer (Holyoak 1991, p. 309), utilisent des stratégies variées pour améliorer leurs performances et modifient ces stratégies en fonction des erreurs identifiées (Chase et Ericsson 1981 ; VanLehn 1991, cité par Ericsson, Krampe et Tesch-Romer 1993, p. 367). Enfin, les experts possèdent des habiletés supérieures dans la façon d'utiliser leur mémoire ${ }^{4}$ ainsi que dans la 
façon de s'autoréguler (Glaser et Chi 1988). En d'autres mots, 1'expert est capable de faire la bonne chose au bon moment (Dorner et Scholkopf 1991, cité par Holyoak 1991,p. 309).

En musique, l'expertise est acquise en travaillant quotidiennement à l'instrument, et l'efficacité des séances de travail est conditionnée par les habiletés métacognitives mises en place par le musicien. C'est d'ailleurs ce qui amène Hallam (2001) à affirmer que le musicien doit posséder des habiletés métacognitives pour effectuer des séances de travail efficaces

afin d'être capable de reconnaître la nature et les exigences d'une tâche spécifique ; d'identifier des difficultés particulières ; de posséder un éventail de stratégies pour faire face à ces difficultés ; de savoir quelle stratégie est appropriée pour réaliser chaque tâche ; de surveiller le progrès menant au but. Si le progrès n'est pas satisfaisant, il faut le reconnaître et utiliser des stratégies alternatives; évaluer les résultats de l'apprentissage dans des contextes de performance et prendre les mesures nécessaires afin d'améliorer la performance dans le futur (ibid., p. 28) ${ }^{5}$.

Toujours selon Hallam, les habiletés métacognitives du musicien doivent inclure également des outils lui permettant de bien gérer le temps de répétition afin d'être capable, entre autres choses, de respecter certains délais professionnels ou de maintenir son niveau de concentration et de motivation. Autrement dit, les habiletés métacognitives seraient nécessaires, voire essentielles, à l'optimisation des répétitions individuelles du musicien. Par ailleurs, les recherches menées par Hallam indiquent que les musiciens experts possèdent davantage d'habiletés métacognitives que les novices, et que ces habiletés sont observables dans la manière dont ils s'exercent pendant le travail instrumental. Ainsi, Hallam (ibid., p. 30-34) explique que les musiciens experts ayant développé des habiletés métacognitives

- $\quad$ sont conscients de leurs propres forces et faiblesses (connaissances sur les personnes $)^{6}$, ce qui leur permet d'identifier, pendant la lecture du nouveau répertoire, les passages nécessitant plus de travail lors des séances subséquentes (activités de planification);

- possèdent de vastes connaissances concernant la nature des différentes tâches à effectuer - lire du nouveau répertoire, organiser la répétition, travailler les passages difficiles, maintenir la concentration, préparer la performance - ce qui serait nécessaire pour les réaliser avec succès (connaissances sur les tâches) ;

$5 \quad$ " [...] in order to be able to recognise the nature and requirements of the particular task; identify particular difficulties; have knowledge of a range of strategies for dealing with these problems; know which strategy is appropriate for tackling each task; monitor progress towards he goal, if progress is unsatisfactory acknowledge this and draw on alternative strategies; evaluate learning outcomes in performance contexts and take action as necessary to improve performance in the future " (Hallam 2001, p. 28).

6 L'identification des habiletés mises entre parenthèses par les auteurs de l'article vise à spécifier les composantes de la métacognition dont il est question dans le texte de Hallam (2001). 
- possèdent une variété de stratégies pouvant être utilisées en fonction de leurs besoins spécifiques et de la tâche à accomplir (connaissances sur les stratégies);

- orientent leur travail en fonction de buts spécifiques (activités de planification);

- possèdent une base de connaissances leur permettant d'évaluer les exigences de la tâche, d'identifier les difficultés (connaissances sur les tâches), de reconnaître leurs erreurs, d'évaluer les progrès (activités de contrôle) et de prendre les mesures nécessaires pour surmonter les problèmes (activités de régulation).

Cela dit, on peut supposer que le professeur de musique, étant lui-même musicien expert, peut aider l'élève à développer de telles habiletés métacognitives afin qu'il puisse effectuer des séances de travail de plus en plus efficaces à l'instrument. Pour aller dans cette direction, il s'avère toutefois important de posséder certaines qualités pédagogiques spécifiques au développement des habiletés métacognitives de l'élève, et que l'enseignant intègre dans son acte pédagogique des stratégies favorisant leur acquisition.

\section{Qualités de l'enseignant-guide de la métacognition}

Les recherches portant sur le développement de la métacognition menées par Lafortune, Jacob et Hébert (2000, p. 53-54) démontrent que, pour favoriser le développement des habiletés métacognitives de l'élève, le professeur doit structurer sa démarche pédagogique en conséquence et posséder également des qualités pédagogiques spécifiques. Il doit premièrement connaître les fondements psychologiques liés à la métacognition ${ }^{7}$ et élaborer des stratégies d'enseignement conçues explicitement pour la développer. Il doit aussi proposer régulièrement à l'élève des moments de réflexion afin de favoriser l'émergence de commentaires métacognitifs et de susciter la verbalisation de ses démarches mentales. Enfin, il doit être en mesure de reconnaître les « déclics métacognitifs » de l'apprenant afin de susciter chez lui une prise de conscience.

De son côté, Doly (1997, p. 61) affirme que l'expert doit éviter de surcharger cognitivement l'élève durant son apprentissage. Pour ce faire, la tâche à réaliser

doit être suffisamment simple pour permettre au sujet de partir de ce qu'il sait, de ses connaissances et compétences actuelles et embryonnaires, mais en même temps suffisamment difficile pour présenter un problème au sujet, qui l'oblige à prendre des risques pour aller au-delà de ce qu'il sait faire tout seul (ibid., p. 59).

L'expert doit aussi savoir utiliser de façon positive les erreurs effectuées par l'élève. Pour aller dans cette direction, il peut aider l'apprenant à les identifier et à les analyser dans le but de l'amener à " prendre conscience des procédures et connaissances qu'il

$7 \quad$ C'est d'ailleurs ce qui nous a motivé à expliquer et regrouper dans la première section de l'article des connaissances liées à la métacognition issues de différentes sources scientifiques. 
a spontanément utilisées et qui ont conduit à l'erreur, pour lui permettre ensuite de construire des stratégies de remédiation, et éventuellement et plus généralement des stratégies pour vaincre l'échec » (Doly 1997, p. 61-62).

De plus, toujours selon Doly (ibid., p. 62-63), l'enseignant doit proposer des activités qui ont du sens pour l'élève, c'est-à-dire des situations d'apprentissage où il peut se représenter le but à réaliser à l'aide de critères d'évaluation permettant d'orienter son activité. En d'autres termes, l'enseignant doit installer chez l'élève une motivation intrinsèque pour réaliser la tâche, motivation qui sera déterminante pour la réussir.

Enfin, pour l'apprenant pouvant déjà s'autoréguler ou réussir son apprentissage seul, l'enseignant doit éviter de lui fournir une aide supplémentaire car, non seulement une telle aide s'avèrerait inutile pour lui, mais elle pourrait même aller jusqu'à inhiber ses compétences ou les stratégies déjà élaborées ou en voie de l'être (ibid., p. 63).

STRATÉGIES PÉDAGOGIQUES VISANT À DÉVELOPPER LES HABILETÉS MÉTACOGNITIVES DE L'ÉLĖVE

Dans cette section, nous proposons deux stratégies pédagogiques pouvant favoriser le développement des habiletés métacognitives de l'élève musicien. Il s'agit de la stratégie en trois phases et du journal de bord, soit deux approches appropriées pour guider le musicien en formation dans son développement métacognitif.

\section{Stratégie en trois phases}

Lafortune, Jacob et Hébert expliquent que « le développement cognitif et métacognitif est un processus graduel d'intériorisation et de personnalisation grâce aux interactions sociales " (Lafortune, Jacob et Hébert 2000, p. 16). Ce processus d'intériorisation est un acte de réflexion complexe dans lequel l'élève entretient consciemment un dialogue intérieur sur la tâche qu'il exécute. En revanche, ce processus n'est pas automatique et il nécessite la médiation d'un guide expérimenté pour en faire un usage adéquat.

La médiation concerne la manière dont un individu expert dans un domaine organise l'environnement physique et mental d'un autre individu moins expérimenté afin qu'il puisse mieux penser, agir et construire son identité (Doly 1997, p. 45). Cette médiation entre l'expert et l'apprenant s'avère même une condition sine qua non pour que l'élève enclenche le processus d'intériorisation requis pour développer ses habiletés métacognitives. Selon Doly (ibid., p. 55), ce processus d'intériorisation repose sur quatre étapes distinctes :

a) D'abord, l'expert doit posséder les connaissances et les compétences nécessaires à la gestion de la tâche ;

b) Ensuite, ces connaissances et compétences doivent être mises en interaction avec le novice, entre autres à l'aide du langage sous forme d'encouragements, de consignes, d'invitations à s'évaluer ;

c) Puis, le novice doit apprendre à utiliser de plus en plus fréquemment ces connaissances et compétences de façon intentionnelle en présence de l'expert ;

d) Enfin, le novice doit arriver à gérer de façon autonome son activité en l'absence de l'expert. 
Dans un contexte pédagogique, ces étapes correspondent, selon Lafortune, Jacob et Hébert (2000, p. 16-19), au modelage (point a), à la pratique guidée (points b et c), à la pratique autonome (point $\mathrm{d}$ ). Nous avons appliqué la séquence proposée par ces auteurs à l'enseignement instrumental pour construire notre stratégie pédagogique ${ }^{8}$.

\section{Le modelage}

Le modelage correspond à la première étape du processus d'intériorisation. Ici, le professeur offre un modèle à l'élève pour accomplir une tâche à partir de ses connaissances personnelles. Lors de cette phase, le professeur verbalise à haute voix sa démarche mentale en même temps qu'il exécute la tâche. Le but du professeur est de rendre visible son dialogue intérieur en modélisant pour l'élève les opérations mentales qu'il doit mettre en action pour réaliser la tâche adéquatement.

Lors de cette phase, le professeur doit d'abord activer les connaissances antérieures de l'élève s'avérant pertinentes pour exécuter la tâche. Cette approche lui permettra de comparer sa façon de l'effectuer avec celle du professeur. Ensuite, le professeur exprime à haute voix de quelle manière il réalise la tâche ainsi que chacune des pensées qui lui viennent à l'esprit lorsqu'il l'exécute. Par exemple, il verbalise les questions qu'il se pose, les actions qu'il effectue pour surveiller son activité, l'évaluer, adapter sa démarche, etc. En d'autres mots, le professeur ne se contente pas ici de donner seulement un exemple, il se donne lui-même en exemple.

Pour illustrer ce concept pédagogique à partir d'un apprentissage postural en musique, l'enseignant pourrait modéliser pour l'élève la façon d'acquérir une bonne posture à l'instrument en verbalisant tous les éléments dont il doit tenir compte pour y arriver en même temps qu'il exécute l'action. Il exprime également à haute voix les ajustements nécessaires qu'il fait en cours de route pour intégrer la position recherchée à son jeu instrumental, tout en lui expliquant les raisons justifiant ces ajustements.

\section{La pratique guidée}

La pratique guidée correspond aux deuxième et troisième étapes du processus d'intériorisation de Doly (1997, p. 55) que l'élève doit parcourir pour mettre en place une démarche d'apprentissage métacognitive. Ici, le professeur invite l'élève à verbaliser sa façon de procéder tout en se référant à la modélisation qu'il a d'abord effectuée. Ensuite, à l'aide de questions et d'encouragements, le professeur aide l'élève à planifier son activité, il attire son attention sur des aspects importants pour bien exécuter la tâche, puis il l'amène à s'autoévaluer et à se corriger pendant la pratique guidée.

8 L'utilisation de cette stratégie doit être favorisée avec des enfants en âge scolaire. Elle peut être utilisée autant en enseignement individuel qu'en groupe. Toutefois, pour l'enseignement en groupe, le professeur peut ajouter une phase - telle que proposé par Lafortune, Jacob et Hébert (2000, p. 18) - entre la pratique guidée et la pratique autonome, soit la pratique coopérative. Lors de cette phase, l'enseignant invite les élèves à se placer en dyades afin de susciter l'échange entre eux concernant leurs façons de faire durant leurs activités d'apprentissage. 
Si on reprend l'exemple postural précédent, lors de cette phase, le professeur inviterait l'élève à verbaliser son dialogue intérieur lorsqu'il cherche à appliquer à son jeu instrumental l'attitude posturale d'abord modélisée devant lui. Lorsque le professeur s'aperçoit que certains éléments n'ont pas été pris en considération par l'élève, il l'encourage et lui pose des questions afin d'attirer spécifiquement son attention sur ces éléments. Le professeur peut également répéter la phase de modelage afin de rendre plus évidents les éléments nécessitant plus d'attention de la part de l'élève avant de continuer la pratique guidée.

\section{La pratique autonome}

La pratique autonome correspond à la dernière étape du processus d'intériorisation de Doly (1997, p. 55), soit celle où l'élève s'exerce sans la présence de l'expert. En principe, lors de cette phase, il doit être en mesure d'entretenir un dialogue intérieur lorsqu'il gère la tâche, ainsi que de se poser des questions pertinentes pour la réaliser adéquatement. En fait, il reproduit l'interaction qui a eu lieu entre lui et l'expert durant la pratique guidée, mais en jouant simultanément cette fois-ci le rôle de l'expert et de l'élève.

Si on reprend l'apprentissage postural donné en exemple lors des deux phases précédentes, l'élève devrait ici être en mesure d'intégrer à son jeu instrumental l'attitude posturale souhaitée, de s'autoévaluer et de mettre en action certains modes d'évaluation lui permettant de se corriger par lui-même si certains problèmes persistent.

\section{Le journal de bord}

La deuxième stratégie que nous proposons pour développer les habiletés métacognitives de l'élève est de réaliser un journal de bord ${ }^{9}$ dans lequel l'élève écrit ses observations au sujet de ses connaissances et de ses habiletés métacognitives. En utilisant l'écriture comme moyen de réflexion, l'élève pose alors un regard sur sa pensée intérieure et laisse des traces qui lui permettent, par la suite, d'évaluer ses progrès et sa manière d'apprendre (Lafortune, Jacob et Hébert 2000, p. 65-72). Par contre, l'action d'écrire ne développe pas automatiquement des habiletés métacognitives. Pour y arriver, le professeur doit guider les observations de l'élève de façon structurée tout en poursuivant des objectifs spécifiques. En d'autres termes, le professeur doit « lui proposer des activités d'écriture qui suscitent des prises de conscience sur la gestion et la régulation de la démarche de ses apprentissages » (ibid., p. 71).

Dans le but d'avoir en main des références sur la manière de guider le processus de réflexion de l'élève, nous avons adapté le questionnaire sur les Connaissances méta-

9 La réalisation d'un journal de bord est une stratégie fréquemment présentée dans la littérature portant sur le développement de la métacognition. Il s'agit d'une approche pédagogique particulièrement adaptée pour favoriser une démarche réflexive chez l'élève. 
cognitives et gestion de l'activité mentale (COMÉGAM) conçu et validé par Richer et al. (2004), aux besoins de la pratique instrumentale. Élaboré pour mesurer et développer la métacognition, ce questionnaire (annexe A), peut aussi guider l'acte pédagogique du professeur d'instrument qui désire amener l'élève à développer ses habiletés métacognitives. Il comporte des énoncés relatifs aux connaissances métacognitives (sur soi, sur la tâche, sur les stratégies) et à la gestion mentale (planification, régulation, contrôle) dans un contexte de répétition instrumentale.

Ce questionnaire comprend 36 énoncés et se divise en six sections. Chaque section porte sur un aspect spécifique de la métacognition. Puisque les énoncés de la version originale étaient conçus pour l'apprentissage en milieu scolaire ${ }^{10}$, nous avons modifié les énoncés du questionnaire original afin qu'il soit adapté au contexte de l'apprentissage instrumental. À titre d'exemple, dans la version originale, le premier énoncé mentionnait : "Je connais les stratégies qui m'aident le plus à apprendre ". Nous avons modifié et adapté ce même énoncé par : "Je connais les stratégies qui m'aident à mieux répéter ».

Une fois que le professeur d'instrument a identifié à l'aide du questionnaire les composantes sur lesquelles l'élève doit se concentrer pour développer ses habiletés métacognitives, il peut ensuite utiliser des fiches de réflexion ${ }^{11}$ (annexe B) pour guider les observations que l'apprenant aura à noter dans son journal de bord. Ici aussi, nous avons modifié les énoncés des fiches de réflexion que l'on retrouve dans la version originale afin qu'ils soient mieux adaptés au contexte de la répétition instrumentale. Chaque fiche vise à faire réfléchir l'élève au sujet d'une composante métacognitive spécifique. Elles sont composées principalement de questions et de pistes de réflexion pour guider l'élève musicien dans sa démarche métacognitive. Nous proposons au professeur d'instrument d'utiliser ce questionnaire et ces fiches de réflexion à l'aide de la procédure suivante :

- En début d'année, l'élève répond au questionnaire afin d'identifier quelles sont les composantes métacognitives qui méritent d'être développées davantage ;

- Tout au long de l'année, le professeur guide l'élève dans ses observations à l'aide des fiches de réflexion. L'élève décrit ensuite ses observations dans son journal de bord ;

- Le professeur invite l'élève à partager ses réflexions afin de lui fournir une rétroaction pertinente à ce sujet, et dans le but de l'aider à développer ses habiletés métacognitives et à améliorer ses séances de répétition.

La procédure expliquée plus haut vise principalement à amener l'élève à réfléchir sur ses séances de répétition instrumentale et à les rendre plus efficaces. Toutefois, les fiches de réflexion peuvent aussi être utilisées durant les leçons et être

10 Cet outil a été développé dans le cadre d'une recherche collaborative visant à rendre autonomes des étudiants de niveau collégial.

11 Ces fiches de réflexion sont en fait intégrées au questionnaire, et leur version originale fût également validée par leurs auteurs (Richer et al. 2004). 
choisies en fonction d'une pièce que l'élève travaille avec son professeur. À titre d'exemple, si un élève guitariste doit apprendre un passage comportant un motif d'arpège devant être joué rapidement, le professeur peut se référer à la fiche 1 portant sur les stratégies et l'adapter, comme nous le suggérons ci-dessous, à la difficulté concernée :

- L'énoncé original «Y a-t-il des actions qui pourraient être posées avant de commencer à répéter? " peut être adapté ainsi : "Y a-t-il des actions qui pourraient être posées avant de commencer à répéter le passage ayant ce motif d'arpège?»;

- L'énoncé original «Y a-t-il une façon de faire qui pourrait t'aider au cours de ta répétition instrumentale ? " peut être modifié dans ce cas-ci par « Y a-t-il une façon de faire qui pourrait t'aider, pendant ta répétition instrumentale, à bien réussir le passage ayant ce motif d'arpège plus rapidement? »;

- Enfin, l'énoncé original « Pour toi, y a-t-il des moments qui sont plus propices pour répéter? » peut devenir " Pour toi, y a-t-il des moments qui sont plus propices pour répéter la vitesse exigée à la main droite pour jouer ce motif d'arpège correctement? ${ }^{12}$.

\section{CONCLUSION}

Selon la littérature spécialisée, l'expert musicien possèderait davantage d'habiletés métacognitives que le musicien en formation. En effet, l'expert démontre une capacité d'analyse lui permettant d'identifier plus facilement et rapidement les problèmes à régler, tandis que le musicien moins expérimenté, notamment celui en début d'apprentissage, aurait plutôt tendance à s'exercer de façon répétitive sans porter un regard réflectif sur ses erreurs et sur les stratégies pouvant les corriger. En revanche, si le professeur d'instrument procure un accompagnement pédagogique approprié, il peut améliorer les habiletés métacognitives de l'élève, ce qui aurait un effet positif sur la qualité de ses séances de travail personnelles.

C'est d'ailleurs ce qui a motivé la rédaction de cet article, car nous voulions exposer au professeur d'instrument les connaissances requises pour travailler plus efficacement dans cette direction, ainsi qu'à lui proposer une adaptation d'outils validés scientifiquement pour seconder son acte pédagogique sur le plan métacognitif. Pour ce faire, nous avons d'abord expliqué les différentes composantes liées à la métacognition, puis proposé deux approches pédagogiques pouvant guider le professeur d'instrument dans le développement des habiletés métacognitives de l'élève. Nous avons également adapté à l'enseignement instrumental un questionnaire et des fiches de réflexion déjà

12 Savoir écrire est une habileté indispensable à l'utilisation de cette stratégie, autant chez le professeur que chez l'élève. Cette stratégie peut servir en enseignement individuel et en groupe. Pour l'enseignement en groupe, chaque élève réalise son propre journal de bord. Par la suite, le professeur invite les élèves à se placer en petits groupes afin de leur faire partager leurs réflexions. Pendant qu'un des élèves expose ses réflexions, les autres font des comparaisons entre leurs propres réflexions et celles de leur collègue. Finalement, le professeur fait un retour sur l'activité avec tout le groupe. 
présents dans la littérature scientifique. Le questionnaire vise à identifier les forces et les faiblesses métacognitives de l'élève, alors que les fiches de réflexion ont pour objectif de guider efficacement l'élève dans sa démarche métacognitive.

L'utilisation de stratégies favorisant le développement de la métacognition dans un contexte d'enseignement instrumental individuel s'avère essentielle pour optimiser l'efficacité des séances de répétition de l'apprenant. Nous croyons que les outils et les approches pédagogiques proposés dans le cadre de cet article peuvent aider le professeur d'instrument à développer plus efficacement les habiletés métacognitives de leurs élèves. Ce questionnaire et ces fiches de réflexion adaptés à l'enseignement instrumental pourraient même être repris pour réaliser des projets de recherche dans ce domaine afin d'en évaluer l'efficacité réelle sur le développement métacognitif de l'élève musicien. Chose certaine, il s'avèrerait essentiel de poursuivre les recherches dans ce domaine, car l'acquisition d'habiletés métacognitives est une composante majeure pour améliorer la qualité des séances de répétition de l'élève musicien.

\section{BIBLIOGRAPHIE}

Aiello, Rita, et Aaron Williamon (2002), " Memory », dans Richard Parmcutt et Gary E. McPherson (dir.), The Science and Psychology of Music Performance. Creative Strategies for Teaching and Learning, Oxford, Oxford University Press, p. 167-181.

Barry, Nancy H., et Susan Hallam (2002), « Practice », dans Richard Parncutt and Gary E. McPherson (dir.), The Science and Psychology of Music Performance. Creative Strategies for Teaching and Learning, Oxford, Oxford University Press, p. 151-165.

Brown, Ann L. (1987), "Metacognition, Executive Control, Self-Regulation and Other More Mysterious Mechanisms ", dans Franz E. Weinert et Rainer H. Kluwe (dir.), Metacognition, Motivation and Understanding, Hillsdale, NJ, Lawrence Erlbam Associates, p. 65-116.

Chase, William G., et Herbert A. Simon (1973), "The Mind's Eye in Chess », dans William G. Chase (dir.), In Visual Information Processing, New York, Academic Press, p. 215-281.

Doly, Anne-Marie (1997), Métacognition et médiation, Auvergne, CDPR.

Dubé, Francis (2006), «Les repères microstructuraux dans l'apprentissage mnémonique de partitions de piano ", thèse de doctorat, Université Laval.

Ericsson, Karl A., Krampe, Ralf Th., et Clemens Tesch-Römer (1993), « The Role of Deliberate Practice in the Acquisition of Expert Performance ", Psychological Review, vol. 100, no 3, p. 363406.

Flavell, John H. (1971), «First Discussants Comments. What is Memory Development the Development of? ", Human Development, vol. 14, n 4, p. 272-278.

Flavell, John H. (1976), « Metacognitive Aspects of Problem-Solving », dans Lauren B. Resnick (dir.), The Nature of Intelligence, Hillsdale, NJ, Lawrence Erlbaum Associates, p. 231-235.

Flavell, John H. (1979), "Metacognition and Cognitive Monitoring. A New Area of CognitiveDevelopmental Inquiry ", American Psychologist, vol. 34, no 10, p. 906-911.

Flavell, John H. (1987), « Speculations About the Nature and Developement of Metacognition », dans Franz E. Weinert et Rainer H. Kluwe (dir.), Metacognition, Motivation and Understanding, Hillsdale, NJ, Lawrence Erlbam Associates, p. 21-29.

Glaser, Robert, et Michelene T. H. Chi (1988), « Overview », dans Michelene T. H. Chi, Robert Glaser et Marshall J. Farr (dir.), The Nature of Expertise, Hillsdale, NJ, Lawrence Erlbaum Associates, p. $15-28$. 
Hallam, Susan (2001), "The Development of Metacognition in Musicians. Implications for Education ", British Journal of Music Education, vol. 18, n 1, p. 27-39.

Holyoak, Keith. J. (1991), "Symbolic Connectionism. Towards Third-Generation Theories of Expertise ", dans Karl A. Ericsson et Jacqui Smith (dir.), Toward a General Theory of Expertise. Prospects and Limits, Cambridge, Cambridge University Press, p. 301-335.

Jørgensen, Harald. (2004), "Strategies for Individual Practice », dans Aaron Williamon (dir.), Musical Excellence. Strategies and Techniques to Enhance Performance, New York, Oxford University Press, p. 85-103.

Lafortune, Louise, Jacob, Suzanne, et Danièle Hébert (2000), Pour guider la métacognition, Québec, Presses de l'Université du Québec.

McPherson, Gary E., et James M. Renwick (2001), « A Longitudinal Study of Self-Regulation in Children's Musical Practice», Music Education Research, vol. 3, n² 2, p. 169-186.

Richer, Jeanne, et al. (2004), "Outil d'évaluation de la métacognition. Processus de validation et utilisation à des fins pédagogiques ", dans Richard Pallascio, Marie-France Daniel et Louise Lafortune (dir.), Pensée et réflexivité. Théories et pratiques, Québec, Presses de 1'Université du Québec, p. 73-106.

Saint-Pierre, Lise (1994), "La métacognition, qu'en est-il ? », Revue des sciences de l'éducation, vol. 20, $\mathrm{n}^{\circ} 3$, p. $529-545$.

Woody, Robert H. (2001), « Learning From the Experts. Applying Research in Expert Performance to Music Education", Applications of Research in Music Education, vol. 19, n 2, p. 9-14.

\section{ANNEXES}

Annexe A : Questionnaire comÉGAM adapté à la répétition instrumentale.

Annexe B : Fiches de réflexion. 